\title{
"This Seems to Work": Designing Technological Systems with The Algorithmic Imaginations of Those Who Labor
}

\author{
Lindsey Cameron \\ Wharton School, University of \\ Pennsylvania \\ Tawanna Dillahunt \\ School of Information, University of \\ Michigan \\ Rida Qadri \\ Urban Information Systems, MIT
}

\author{
Angèle Christin \\ Department of Communication, \\ Stanford University \\ Madeleine C. Elish \\ Google Research
}

\author{
Noopur Raval \\ AI Now Institute, New York \\ University \\ Elizabeth Anne Watkins \\ Center for Information Technology \\ Policy, Princeton University
}

\author{
Michael Ann Devito \\ School of Communication, \\ Northwestern University \\ Mary L. Gray \\ Microsoft Research
}

\author{
Melissa Valentine \\ Center for Work, Technology, and \\ Organization, Stanford University
}

\begin{abstract}
Algorithmically mediated systems and tools are used by workers across the globe. Many of these workers are in low-power positions, where they have little leverage to make demands around transparency, explanation, or terms of use, yet, at the same time rely deeply on these systems for many aspects of their jobs. This tension between little power and high reliance drives the production of intensive algorithmic imaginaries, where workers engage in meaning-making to construct understandings of these systems. Yet, there has been little attention paid to the diversity and ingenuity of algorithmic understandings crafted by the workers. In this workshop, our goal is to bring together researchers and practitioners from across disciplines to create a research agenda, compare vocabularies, and discuss methodologies around this form of "folk tradecraft." This toolkit will help elicit insights into these phenomena and ultimately build mechanisms by which the labor of algorithmic meaning-making can be respected, understood, and leveraged for system design.
\end{abstract}

\section{CCS CONCEPTS}

- Human-centered computing; Human computer interaction (HCI); HCI theory, concepts and models; - Humancentered computing; Human computer interaction (HCI); HCI design and evaluation methods; User studies;

Permission to make digital or hard copies of part or all of this work for personal or classroom use is granted without fee provided that copies are not made or distributed for profit or commercial advantage and that copies bear this notice and the full citation on the first page. Copyrights for third-party components of this work must be honored. For all other uses, contact the owner/author(s).

CHI '21 Extended Abstracts, May 08-13, 2021, Yokohama, Japan

(c) 2021 Copyright held by the owner/author(s).

ACM ISBN 978-1-4503-8095-9/21/05.

https://doi.org/10.1145/3411763.3441331

\section{KEYWORDS}

Gig work, algorithm, sociotechnical explanation ;work, platform, algorithmic imaginary

ACM Reference Format:

Lindsey Cameron, Angèle Christin, Michael Ann Devito, Tawanna Dillahunt, Madeleine C. Elish, Mary L. Gray, Rida Qadri, Noopur Raval, Melissa Valentine, and Elizabeth Anne Watkins. 2021. "This Seems to Work": Designing Technological Systems with The Algorithmic Imaginations of Those Who Labor. In CHI Conference on Human Factors in Computing Systems Extended Abstracts (CHI '21 Extended Abstracts), May 08-13, 2021, Yokohama, Japan. ACM, New York, NY, USA, 5 pages. https://doi.org/10.1145/3411763.3441331

\section{BACKGROUND}

The CHI community has begun to build a foundation to study the strategies, tactics, and methods utilized by users of algorithmic and AI-infused systems. There is burgeoning research on how algorithmic understandings from non-expert users hold implications for the design of technological platforms such as recommender systems [1], social media feeds $[5,8,9]$, data-driven decision support in medicine [6] and computer security [3, 17]. However, most of this work has either focused on white-collar and professional workers, or end consumers of these systems.

There has been little attention paid to the diversity of imaginaries [4], meaning-making [7], and ingenuity of understandings crafted by the workers within algorithmically-mediated systems traditionally considered 'low-power' such as ridehail drivers, delivery personnel, and micro-task workers. Contemporary studies [7, 12$14,16,18]$ have found consistent evidence of these workers doing their own forms of research to reverse engineer the algorithms, including modifying their own behaviour to create a platform environment better optimized for the worker, ultimately producing a form of "folk tradecraft" [19]. A concurrent discussion is also developing, examining the processes underlying community-based building of technical expertise among people who work on socialmedia platforms, for example beauty vloggers' "algorithmic gossip" 
[2]. The tension between low levels of power in these sociotechnical systems (i.e., little leverage to demand explanation or determine whether algorithmic tools are integrated into their work) and reliance on these systems (i.e. being in a position where they deeply rely on these systems for every aspect of their jobs) drives the production of intensive algorithmic imaginaries. We contend that this cognitive labor of understanding algorithmic systems, and resulting decisions around ad-hoc tactics, are skills that need to be respected, understood, and leveraged into the design of platforms themselves $[10,12]$.

This workshop focuses primarily on the imaginaries of lowpower workers in algorithmically mediated tech platforms and algorithmic systems. While our concern is largely with tech platforms and workers such as ride-hail drivers, delivery workers, micro-task workers, and at-home healthcare workers, we are also concerned with the invisible impacts these integrations have on low power workers in other spaces, such as nurses[11] and grocery-store workers [15]. At heart, our goal is to build a research agenda examining how the integration of algorithms fosters a tension between low levels of end worker power in these systems and workers' extreme reliance on these systems: the outcome of which is rich imaginative labor strategically deployed. Our focus is less on design challenges for skilled professionals in specific work contexts or human-automation collaboration where humans integrate algorithmic outputs into complex existing workflows. We encourage those interested in these arenas to seek out a complementary CHI workshop, Automation Experience at the Workplace.

We welcome the chance to elevate the ways $\mathrm{CHI}$ community talks about low-power workers who are managed by or engage with algorithmic platforms, with the explicit goal of centering workers and their agency. The aims of this workshop are to foster a cohesive discourse across the siloes of sociology, organizational theory, communications studies, anthropology, and $\mathrm{HCI}$, and produce a collection of perspectives on:

- Imaginations of what technology design would look like if it respected worker agency and leveraged their understandings of algorithmic underpinnings;

- New ways of framing the interactions between on-ground algorithmically-mediated workers and the technological system;

- Innovative methodological breakthroughs which can provide better insight into the 'people's research' workers undertake, their mental models of algorithmic systems and/or their influence on how the platform functions.

\section{ORGANIZERS}

\subsection{LINDSEY CAMERON}

Lindsey is an assistant professor of management at the Wharton School, University of Pennsylvania. Her research focuses on how changes in the modern workplace (e.g., algorithms/machine learning, short-term employment contracts, variable pay) affect work and workers. Her recent work examines how algorithms are fundamentally reshaping the nature of managerial control and how workers navigate this new workplace. Her work has been published in the journals Organizational Behavior and Human Decision Process, Annual Review of Organizational Psychology and Organizational
Behavior, and proceedings of the Association of Computing Machinery.

\subsection{ANGÈLE CHRISTIN}

Angèle Christin is an assistant professor in the Department of Communication and affiliated faculty in the Sociology Department and Program in Science, Technology, and Society at Stanford University. She studies how algorithms and analytics transform professional values, expertise, and work practices. Her book, Metrics at Work: Journalism and the Contested Meaning of Algorithms (Princeton University Press) focuses on the case of web journalism, analyzing the growing importance of audience data in web newsrooms in the U.S. and France. In a new project, she examines the paradoxes of algorithmic labor through a study of influencers on YouTube, Instagram, and TikTok. Angèle received her $\mathrm{PhD}$ in Sociology from Princeton University and the EHESS (Paris). She is an affiliate at the Data \& Society Research Institute and a Visiting Researcher at the Social Media Collective at Microsoft Research New England.

\subsection{MICHAEL ANN DEVITO}

Michael Ann DeVito (she/her) is an interdisciplinary social/behavioral scientist based in Human/Computer Interaction and Social Computing. Currently a doctoral candidate in the Media, Technology, and Society program at Northwestern University's School of Communication, she is also a Cognitive Science specialist, and works as a graduate researcher in Northwestern's Social Media Lab. Her research centers around how humans adapt to the complex algorithmically-driven systems that increasingly surround us, including how we come to understand these systems, and how they affect major social processes such as the formation and presentation of the self-concept. She has published extensively at $\mathrm{CHI}$ and $\mathrm{CSCW}$, among other venues.

\subsection{TAWANNA DILLAHUNT}

Tawanna Dillahunt, $\mathrm{PhD}$. is an Associate Professor at the University of Michigan's School of Information and holds a courtesy appointment with the Electrical Engineering and Computer Science departments. Working at the intersection of human-computer interaction; environmental, economic, and social sustainability; and equity, her research investigates and implements technologies to support the needs of populations who have been historically excluded or left out. She and her team have developed digital employment tools that address the needs of job seekers with limited digital literacy and education; assessed real-time ridesharing and online grocery delivery applications among lower-income and transportation-scarce groups, and proposed models for novice entrepreneurs to build their technical capacity.

\subsection{MADELEINE C. ELISH}

Madeleine Clare Elish is a cultural anthropologist whose work examines the social impacts of AI and automation on society. She recently joined Google as a Senior Research Scientist working on the Ethical AI team. Previously, she co-founded and led the AI on the Ground Initiative at Data \& Society Research Institute, which uses social science research to inform future design, use, and governance of AI systems. She has conducted field work across varied industries 
and communities, ranging from the Air Force, the driverless car industry, and commercial aviation to precision agriculture and emergency healthcare. Her research has been published and cited in scholarly journals as well as publications including The New York Times, Slate, The Guardian, Vice, and USA Today. She holds a $\mathrm{PhD}$ in Anthropology from Columbia University and an S.M. in Comparative Media Studies from MIT.

\subsection{MARY GRAY}

Mary L. Gray is a Senior Principal Researcher at Microsoft Research as well as a Berkman Klein Center for Internet and Society Faculty Associate at Harvard University. Mary also maintains a faculty position in the School of Informatics, Computing, and Engineering with affiliations in Anthropology and Gender Studies at Indiana University. Mary, an anthropologist and media scholar by training, focuses on how everyday uses of technologies transform people's lives. In 2020, Mary was named a MacArthur Fellow for her contributions to anthropology and the study of technology, digital economies, and society.

\subsection{RIDA QADRI}

Rida is a PhD Candidate in Urban Information Systems at Massachusetts Institute of Technology studying the domestication of globalizing technologies in non-western urban spaces, with a focus on digitization of work. Her dissertation project uses the case of mobility platform drivers in Jakarta to highlight the agentic algorithmic understandings and strategies of resistance crafted by gig workers. By showcasing the importance of market institutions, worker knowledge and social relationships as a scaffolding for digital systems, her research directly lends itself to imagining more inclusive and contextually appropriate forms of digital work. Reflecting her interdisciplinary interests, she has published in and spoken at diverse conference proceedings such as Association of Collegiate Schools of Planning (ACSP), Institute for Global Law and Policy, International Conference on Computers in Urban Planning and Urban Management (CUPUM), AAAI/ACM Conference on AI, Ethics, and Society.

\subsection{NOOPUR RAVAL}

Noopur Raval is a postdoctoral research fellow at the AINow Institute at New York University (from Sept 2020) and a CTSP Fellow at UC Berkeley (2020-21). She received her PhD in Informatics from the University of California Irvine in September 2020. Her dissertation titled 'Platform Living - theorizing life, work and ethical enactments in the gig economy' looked at work, consumption and life experiences within gig platforms through ethnographic research in Bengaluru (among other places). It also explored the moral economy of the gig economy. She is also pursuing individual and collaborative research on the deep histories of datafication drawing on the histories and presents of post colonies. She was a Technology, Law and Society Fellow (2017-18) at UC Irvine and is an alumna of the Berkman-Klein Center for Internet \& Society at Harvard University (2016-17).

\subsection{MELISSA VALENTINE}

Melissa Valentine is an Assistant Professor of Management Science at Stanford University, where she co-directs the Center for Work, Technology, and Organization. Her research focuses on understanding how work and organizations are changing as a result of crowdsourcing, data, and algorithms. She conducts in-depth observational studies that detail how work, organizations, and technologies change over time. Melissa has received several best paper awards for research and has been recognized with an NSF CAREER award. Melissa holds a bachelor's degree from Stanford University, a master's degree from NYU, and a Ph.D. from Harvard University.

\subsection{ELIZABETH ANNE WATKINS}

Elizabeth Anne Watkins is a Postdoctoral Research Associate at the Princeton Center for Information Technology Policy, where she studies AI's impact on sociotechnical systems with a focus on the workplace and usable security. She completed her doctorate at Columbia University where she was trained as an organizational sociologist in the field of Communications. She uses qualitative methods to analyze how people interpret, negotiate, and strategize around algorithmic tools in their work. She has published or presented at the conferences on Computer-Human Interaction (CHI), Computer-Supported Cooperative Work (CSCW), Algorithmic Fairness, Accountability, and Transparency (FAccT), and the annual meetings of the Academy of Management (AOM), and the Society for the Social Studies of Science (4S). She holds a Master of Science from MIT and is an affiliate at the Data \& Society Research Institute.

\section{WEBSITE:}

TBD pending acceptance.

\section{PRE-WORKSHOP PLANS}

Organizers have active engagement in Twitter and Slack communities of HCI, sociology and critical data studies scholars. We also plan on circulating the CFP on mailing lists including AOIR (Association of Internet Researchers), AFOG (Algorithmic Fairness and Opacity Group), WAIM (Work in the Age of Intelligent Machines Coordination Group).

\section{WORKSHOP STRUCTURE}

This one-day workshop will comprise a series of moderated discussions and small group brainstorming sessions around themes generated from participants position papers. The end goal would be to cultivate recommendations around theoretical, methodological and design interventions that can be made to respect the algorithmic imaginations of those who labor. We will limit the workshop participants to 15 not including the organizers. Before the workshop we will survey the participants regarding any suggested themes they wish to share and the types of engagements they want to have, to better divide them into breakout rooms. We will also survey all participants on their ideal and less-than-ideal timeslots, to accommodate participants across time zones. All sessions will take place on one organizers' institutional Zoom account, with a dedicated, concurrent Slack channel for all sessions, where participants can interact asynchronously. Recognizing the fatigue which can accompany virtual events, the last two sessions will be optional. 


\subsection{Mapping Themes}

- Given the diverse range of expertise represented by our organizers, we will conduct two one-hour panel discussions around the key themes which have emerged from participant position papers. This panels will feature Lindsey Cameron, Madeleine C. Elish, Mary L. Gray, Melissa Valentine, and Angèle Christin.

\subsection{Brainstorming Interventions}

- 40 minutes breakout session where participants are divided into thematic groups based on position papers to produce interventions to share with the group;

- Each group will have one organizer function as moderators;

\subsection{Generating Recommendations}

- 15-20 minute presentation from each group to share recommendations emerged from their discussions.

- Generate a working document in end of the workshop synthesizing the recommendations for theory, method and design which have emerged from the brainstorming session and group discussion;

- This document will become a position paper and proposal for a special issue or anthology in a book series.

\section{POST-WORKSHOP PLANS}

Sustained coordination and community-building are central goals of this workshop, in particular because we anticipate participants will hail from different disciplinary homes. We will create either a specialized mailing list or Slack group to keep participants in communication with each other and to facilitate a concrete output from this workshop. Some of these outputs will be intangible, including a reading list and cohesive working group to foster continued collaboration. Tangible outputs will include either an edited volume or anthology from a university press, or a special issue of a relevant journal, a compilation of theoretical, methodological and design-focused recommendations, or some combination of these outputs.

\section{CFP}

"This Seems to Work": Designing Technological Systems with The Algorithmic Imaginations of Those Who Labor is a one day workshop to be held during CHI 2021. New scholarship has hinted the ingenuity of workers' mental models of algorithmic systems, especially those traditionally considered 'low-skilled' such as ridehail drivers, delivery personnel, and micro-task workers. This workshop asks how we can respect and leverage this labor of understanding algorithmic systems and the ad-hoc creation of subsequent tactics?

While this workshop invites conversation around any professionals who interact with opaque technological systems, we welcome papers which elevate how the $\mathrm{CHI}$ community talks about lowpower workers managed by or engaging with algorithmic/digital platforms, with the goal of centering workers and their agency.

We invite position papers, from a range of disciplines, which discuss:
Table 1: Workshop Schedule: Sunday, May 9

\begin{tabular}{ll}
\hline Time & Activity \\
\hline $11: 00$ ET & Welcome and organizer introductions \\
$11: 10$ & Panel Discussion 1, Mapping Themes \\
$12: 10$ & Break \\
$12: 25$ & Breakout Session (synchronous in \\
& Zoom): Presentations chaired by \\
& Organizer A \\
& Breakout Session (asynchronous in \\
& Slack): Presentations chaired by \\
& Organizer B \\
& Main Room Session: Brainstorming \\
& Interventions chaired by Organizer C \\
& Break \\
12:55 & Panel Discussion 2, Mapping Themes \\
& Breakout Session*: Generating \\
$13: 20$ & Recommendations; Theory chaired by \\
$13: 50$ & Organizer A \\
$14: 50$ & Breakout Session*: Generating \\
& Recommendations; Methods chaired by \\
& Organizer B \\
& Breakout Session*: Generating \\
& Recommendations; Design chaired by \\
& Organizer C \\
& Networking Breakout Session (optional) \\
& Break \\
& Main Room Session: Next Steps; \\
& Intangible and Tangible Outputs \\
& (optional) \\
\hline $14: 40$ &
\end{tabular}

- Imaginations of what technology design would look like if it leveraged workers understandings of algorithmic underpinnings;

- New ways of framing interactions between on-the-ground algorithmically mediated workers and technological systems;

- Innovative methodological breakthroughs which can provide insight into the 'people's research' workers undertake, i.e. cognitive labor, sensemaking, or algorithmic imaginaries, and/or their influence on platform functions.

Papers will be evaluated based on their fit with the workshop theme. At least one author of an accepted paper must attend the workshop and all participants must register for both the workshop and at least one day of the conference. Interested participants should email papers up to 4 pages in length in the $\mathrm{CHI}$ extended abstract format to thosewholabor@gmail.com no later than February 21 2021 AOE.

\section{REFERENCES}

[1] S. Bishop. 2019. Managing visibility on YouTube through algorithmic gossip. New media \& society, 21 (11-12), pp.2589-2606.

[2] Cristian Bravo-Lillo, Lorrie Faith Cranor, Julie Downs, and Saranga Komanduri. 2011. Bridging the Gap in Computer Security Warnings: A Mental Model Approach. IEEE Security \& Privacy Magazine 9 (2): 18-26. https://doi.org/10.1109/ MSP.2010.198.

[3] Taina Bucher. 2017. The Algorithmic Imaginary: Exploring the Ordinary Affects of Facebook Algorithms. Information, Communication \& Society 20 (1): 30-44. 
https://doi.org/10.1080/1369118X.2016.1154086.

[4] Jenna Burrell, Zoe Kahn, Anne Jonas, and Daniel Griffin. 2019. When Users Control the Algorithms: Values Expressed in Practices on Twitter. Proceedings of the ACM on Human-Computer Interaction, 3 (CSCW), pp.1-20.

[5] Cai, C.J., Winter, S., Steiner, D., Wilcox, L. and Terry, M., 2019. "Hello AI": Uncovering the Onboarding Needs of Medical Practitioners for Human-AI Collaborative Decision-Making. Proceedings of the ACM on Human-Computer Interaction, 3(CSCW), pp.1-24.

[6] Lindsey Cameron . 2020. Allies or Adversaries? Making Meaning of the Gig Employment Relationship. Academy of Management Proceedings 2020 (1): 20302. https://doi.org/10.5465/AMBPP.2020.67.

[7] Angèle Christin. 2020. Metrics at Work: fournalism and the Contested Meaning of Algorithms. Princeton: Princeton University Press.

[8] Michael Ann DeVito, Jeremy Birnholtz, Jeffery T. Hancock, Megan French, and Sunny Liu. 2018."How People Form Folk Theories of Social Media Feeds and What It Means for How We Study Self-Presentation. In Proceedings of the 2018 CHI Conference on Human Factors in Computing Systems - CHI '18, 1-12. Montreal QC, Canada: ACM Press. https://doi.org/10.1145/3173574.3173694.

[9] Tawanna R Dillahunt, Sheena Erete, Roxana Galusca, Aarti Israni, Denise Nacu, and Phoebe Sengers. 2017. Reflections on Design Methods for Underserved Communities. In Companion of the 2017 ACM Conference on Computer Supported Cooperative Work and Social Computing - CSCW'17 Companion, 409-13. Portland, Oregon, USA: ACM Press. https://doi.org/10.1145/3022198.3022664.

[10] Tawanna R Dillahunt, Xinyi Wang, Earnest Wheeler, Hao Fei Cheng, Brent Hecht, and Haiyi Zhu. 2017. The Sharing Economy in Computing: A Systematic Literature Review. Proceedings of the ACM on Human-Computer Interaction 1 (CSCW): 1-26. https://doi.org/10.1145/3134673.
[11] Madeleine C. Elish and Elizabeth Anne Watkins. 2020. Repairing Innovation: A Study of Integrating AI in Clinical Care (New York: Data \& Society Research Institute, 2020), https://datasociety.net/pubs/repairing-innovation.pdf.

[12] Mary L. Gray, and Siddharth Suri. 2019. Ghost Work: How to Stop Silicon Valley from Building a New Global Underclass. Boston: Houghton Mifflin Harcourt.

[13] Katherine C. Kellogg, Melissa A. Valentine, and Angéle Christin. 2020. Algorithms at Work: The New Contested Terrain of Control." Academy of Management Annals 14 (1): 366-410. https://doi.org/10.5465/annals.2018.0174.

[14] Alexandra Mateescu and Madeleine Elish. 2019. AI in context: the labor of integrating new technologies. Data \& Society.

[15] Rida Qadri. 2020. Algorithmized but Not Atomized? How Digital Platforms Engender New Forms of Worker Solidarity in Jakarta. In Proceedings of the AAAI/ACM Conference on AI, Ethics, and Society, 144-144. New York NY USA: ACM. https://doi.org/10.1145/3375627.3375816.

[16] Rick Wash. 2010. Folk Models of Home Computer Security. In Proceedings of the Sixth Symposium on Usable Privacy and Security - SOUPS '10, 1. Redmond, Washington: ACM Press. https://doi.org/10.1145/1837110.1837125.

[17] Elizabeth Anne Watkins, E.A. 2020. Took a Pic and Got Declined, Vexed and Perplexed: Facial Recognition in Algorithmic Management. In Companion of the 2020 ACM Conference on Computer Supported Cooperative Work and Social Computing.

[18] https://doi.org/10.1145/3406865.3418383

[19] Meredith Whittaker. 2020. "The folk tradecraft of workers observing and assessing opaque algorithmic management systems then modifying their behavior in response is both heartbreaking and impressive "people's research." Twitter. Available at https://twitter.com/mer edith/status/1300878248059056134. 